\title{
Surgical Reconstruction for Intrathoracic Aneurysm Originating from Proximal Part of the Right Subclavian Artery
}

\author{
Hiroki Mizoguchi, MD, Takuya Miura, MD, PhD, Kazushige Inoue, MD, PhD, Takashi Iwata, MD, PhD,
}

Keikou Tei, MD and Yuuki Horio, MD

\begin{abstract}
Subclavian artery aneurysms are comparatively rare in peripheral aneurysms. We experienced a case of intrathoracic aneurysm originating from the proximal part of the right subclavian artery. A 78 year-old man was referred to our hospital with the diagnosis of a right subclavian artery aneurysm. Enhanced computed tomography demonstrated an intrathoracic aneurysm, originating from the right subclavian artery just proximal of its origin. Through a median sternotomy and supra-infraclavicular incision, we reconstructed the brachiocephalic and right common carotid arteries and bypassed to the distal part of the right subclavian artery by using a T-shaped vascular graft and the aneurysm was excluded.
\end{abstract}

Keywords: subclavian artery aneurysm, intrathoracic aneurysm, graft replacement

\section{INTRODUCTION}

Sin ubclavian artery aneurysm is a rare aneurysm; its incidence has been reported as $3.5 \%$ of all peripheral aneurysms. ${ }^{1)}$ The surgical procedure and approach depend on the size and location of the aneurysms. ${ }^{2)} \mathrm{We}$ experienced a surgical case of intrathoracic aneurysm originating from the proximal part of the right subclavian artery. We discuss the relation between the location of an aneurysm and the surgical procedure.

\section{CASe Report}

A 78 year-old man, who had gone to the hospital because of hoarseness, was pointed out to have a mediastinal mass by computed tomography (CT). At that time, positron-emission tomography denied the malignancy

Kansai Rosai Hospital, Department of Cardiovascular Surgery, Amagasaki, Hyogo, Japan

Received: October 11, 2011; Accepted: January 21, 2012 Corresponding author: Hiroki Mizoguchi, MD. Kansai Rosai Hospital, Department of Cardiovascular Surgery, 3-1-69 Inabasou, Amagasaki, Hyogo 660-8511, Japan

Tel: +81-6-6416-1221, Fax: +81-6-6419-1870

E-mail: hmizo131@hotmail.co.jp of the mass, and it had been observed. Eight years later, it developed a tendency to increase in size, and he was referred to our hospital for surgery. He had no history of trauma, and no pulsatile mass was found at his supraclavicular fossa on physical examination. A chest radiograph indicated an intrathoracic mass in the right upper thorax. Contrast-enhanced and three-dimensional reconstructed $\mathrm{CT}$ demonstrated an intrathoracic aneurysm, which was $60 \times 58 \mathrm{~mm}$ in size, and it originated from the right subclavian artery just proximal of its origin (Fig. 1A and 1B). Brain magnetic resonance angiography showed complete communication of the circle of Willis; however, the vertebral artery was left dominant (Fig. 1C). Therefore, we monitored the regional saturation of oxygen $\left(\mathrm{rSO}_{2}\right)$ of the brain with the INVOS $5100 \mathrm{C}$ cerebral oximeter (Somanetics, IL, USA) during operation.

We started an operation via median sternotomy and right supraclavicular incision. At first, through a median sternotomy, the brachiocephalic artery, right common carotid artery and right subclavian aneurysm were identified. However, the distal side of the aneurysm was not well approached through these incisions because of dense adhesion between the aneurysm and surrounding tissues. After systemic heparinization, clumping the brachiocephalic artery, the right cerebral clamping $\mathrm{rSO}_{2}$ 

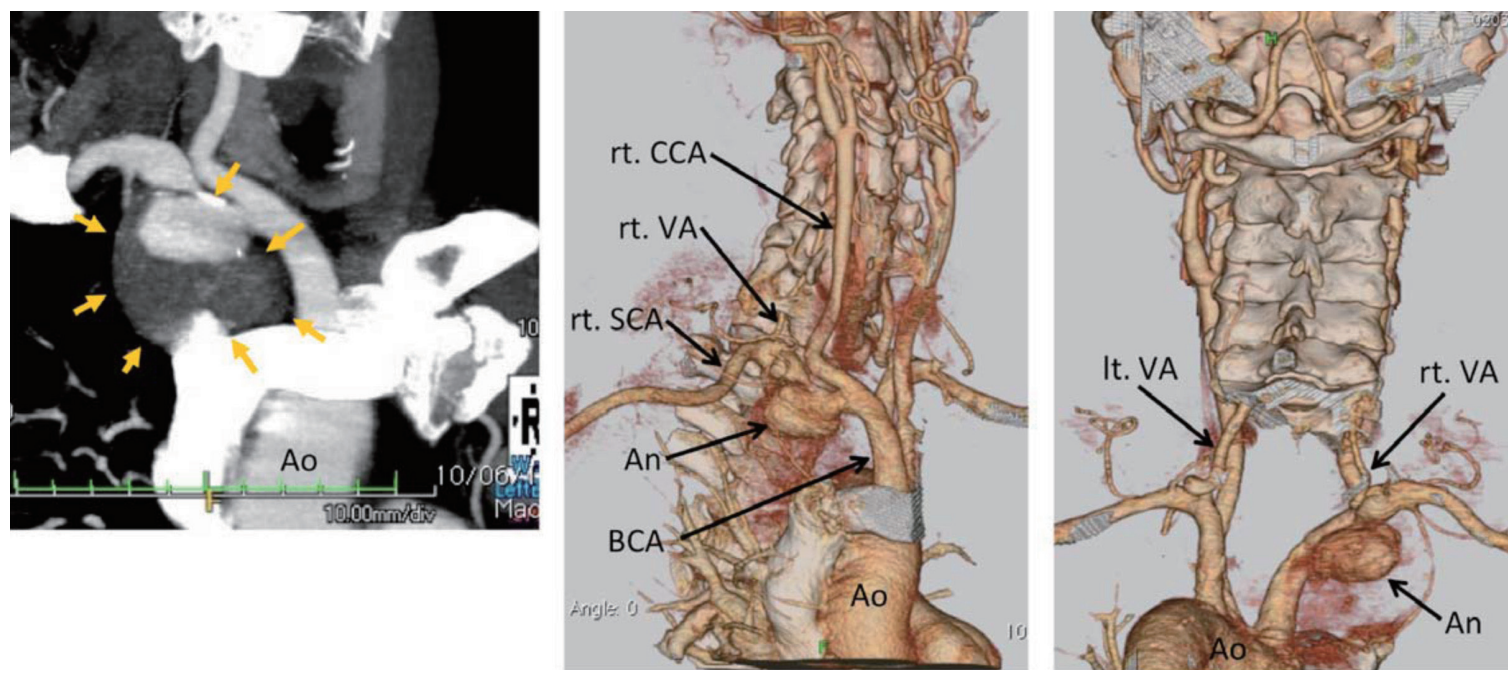

Fig. 1 Preoperative computed tomography (CT) findings.

A: The right anterior oblique view of enhanced CT, the aneurysm was originated from the

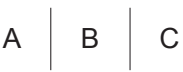
right subclavian artery just proximal of its origin (arrows).

B: The three-dimensional CT (3D-CT) from right anterior oblique view.

C: The 3D-CT from posterior view demonstrated that the vertebral artery was left dominant. Ao: aorta; BCA: brachiocephalic artery; An: aneurysm; rt. SCA: right subclavian artery; rt. VA: right vertebral artery; lt. VA: left vertebral artery; rt. CCA: right common carotid artery

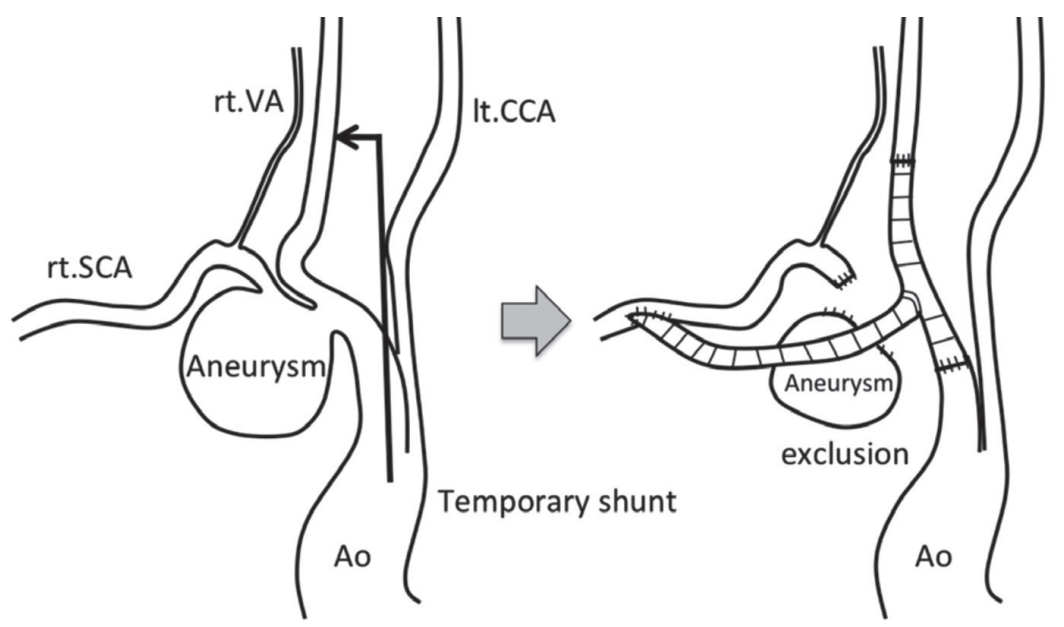

Fig. 2 The schema of operation. We reconstructed the brachiocephalic and right common carotid arteries, and bypassed to the distal part of the right subclavian artery by using a T-shaped vascular graft, and the aneurysm was excluded.

Ao: aorta; rt. SCA: right subclavian artery; rt. VA: right vertebral artery; rt. CCA: right common carotid artery; 1t. CCA: left common carotid artery

dropped; thus, we decided to use a temporary shunt. We inserted a shunt needle into the ascending aorta as an inflow source, and then the right common carotid artery was perfused from this shunt. After this shunting, the brachiocephalic artery was clamped again, and the right cerebral $\mathrm{rSO}_{2}$ was maintained. During this temporary shunt, we reconstructed the brachiocephalic and right common carotid arteries by using a T-shaped knitted Dacron vascular graft through a median sternotomy. Concerning the reconstruction of the right subclavian artery, it was difficult to anastomose in an end-to-end fashion, just distal to the aneurysm; therefore, we added an infraclavicular incision and bypassed the graft to the distal part of the right subclavian artery through this incision. After the complete reconstruction using a Tshaped graft, the aneurysm was excluded, and the right subclavian artery was closed as a stump at just distal side of the aneurysm (Fig. 2).

Postoperatively, the patient achieved complete remission and was discharged from the hospital on day 

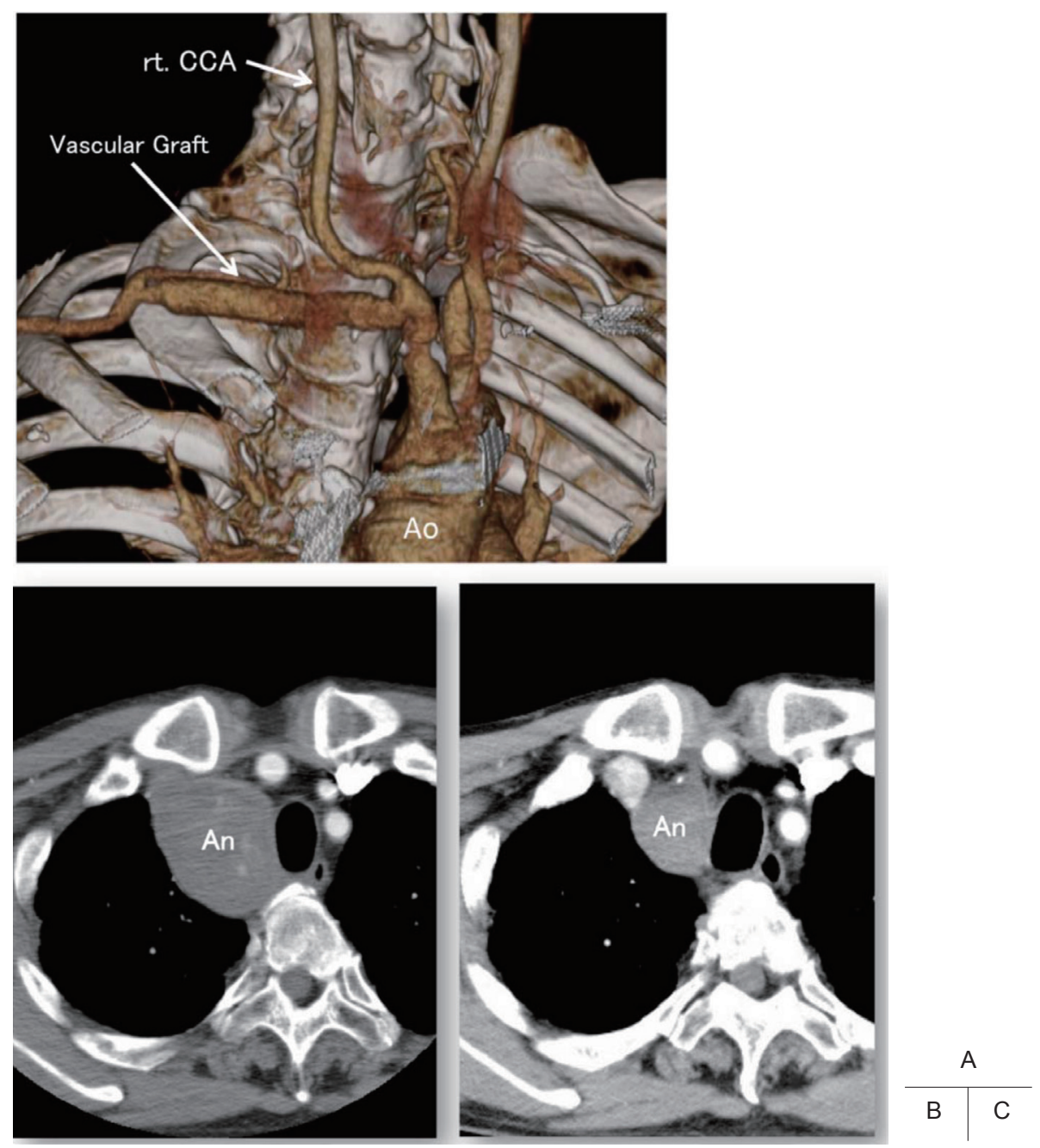

Fig. 3 One year follow up computed tomography.

A: One year follow up three-dimensional computed tomography showed the graft patency.

B: The axial view of preoperative enhanced computed tomography.

C: The axial view of 1 year follow up enhanced computed tomography showed a regression of the excluded aneurysm (compared with Fig. 3B). rt. CCA: right common carotid artery; Ao: aorta; An: aneurysm

21 after the operation. At 1 year of follow up, contrastenhanced CT demonstrated graft patency and reduction in the size of the excluded aneurysm (Fig. 3).

\section{Discussion}

Subclavian artery aneurysms are rare, and there is the possibility of life- and limb-threatening complications, such as thrombosis, embolism, and rupture. The subclavian artery has intra-thoracic and extra-thoracic parts while anatomically, it may be divided into three parts. ${ }^{3)}$ The practical categorization into three parts has been suggested as follows: proximal, middle and distal parts. ${ }^{4)}$ The proximal part extends from the origin, with the brachiocephalic artery on the right side and aorta on the left, to the medial border of the scalene muscles. The middle part of the subclavian artery is located dorsal to the anterior scalene muscles. The distal part of the subclavian artery extends from the lateral border of the anterior scalene muscle to the lateral border of the first rib. ${ }^{4,5)}$ Accordingly, intrathoracic aneurysms are mainly found in the proximal part. The majority (39\%) of subclavian artery aneurysms are located in the proximal segment of the subclavian artery, and the middle and distal segments 
account for $25 \%$ and $24 \%$, respectively. ${ }^{3)}$ Furthermore, thromboembolic complications are mainly related to distal aneurysms, and middle and proximal aneurysms appear to have a tendency to rupture.

An operation should be considered in all cases because the risk of thromboembolic complications is unrelated to the diameter of the aneurysms. ${ }^{3,6)}$ The approach of surgery differs according to the location of the aneurysms. Aneurysms of the distal and middle part of the subclavian artery are easily exposed by a supraclavicular incision. If the surgical view is poor, and further exploration is required, an infraclavicular incision or clavicular division (or resection) may also be used. ${ }^{3,7)}$ Bito et al. ${ }^{7)}$ reported a successful case of exposing the middle part of the subclavian artery with a clavicular division, and concluded that dividing the clavicle is considered useful for achieving an adequate operative field to treat the vessel safely. Repair of these portions is usually achieved by resecting the aneurysm and vascular graft replacement.

The access and procedure for the proximal part of subclavian artery aneurysms is fairly complicated. It is essentially different from whether right or left because the right subclavian artery originates from the brachiocephalic artery and the left subclavian artery originates from the thoracic aortic arch. Proximal aneurysms of the left subclavian artery can be approached through a high, left posterolateral thoracotomy. Moreover, if aneurysms originate from only the ostium of the aorta, a median sternotomy (with left supraclavicular exten$\operatorname{sion}^{8)}$ ) is necessary and aortic arch replacement may be able to be performed. On the other hand, access options for proximal right subclavian artery aneurysms are right thoracotomy or median sternotomy, if necessary, with supra or infraclavicular incisions. Several surgical techniques have been reported for proximal right subclavian artery aneurysms. Currently, it appears that arterial graft interposition or primary end-to-end anastomosis is common subsequent to aneurysmal resection. ${ }^{2)} \mathrm{We}$ reconstructed the brachiocephalic and right common carotid arteries and bypassed to the distal part of the right subclavian artery because of the following reasons: First, the aneurysm extended from the origin of the subclavian artery (the innominate bifurcation); in this situation, it was difficult to reconstruct only the right subclavian artery because the proximal anastomotic neck could not be preserved. Second, the aneurysm was adhesive and large, so the distal anastomosis site of the subclavian artery could not be mobilized to anastomose in an endto-end fashion from sternotomy and supraclavicular sides. Furthermore, separation of aneurysm was risky considering the bleeding. For these reasons, we decided to close as a stump at just distal side of the aneurysm with aneurismal exclusion and bypass to the distal part of the right subclavian artery. By excluding the aneurysm, there was concern about aneurysmal expansion due to the back pressure exerted by several inflow arteries toward the aneurysm. Salo et al. ${ }^{9)}$ recommend aneurysmal exclusion rather than resection; they suggest that exclusion is technically easier than resection and achieves the same postoperative long-term results. Although aneurysmal expansion due to back pressure is a possibility, in our case, enhanced $\mathrm{CT}$ at the 1 year follow-up showed that the excluded aneurysm had regressed. It is important to conduct further clinical follow-ups.

Concerning the reconstruction of the right carotid artery, preoperative assessment of craniocervical arteries by contrast enhanced $\mathrm{CT}$ and magnetic resonance imaging is necessary. In our case, the vertebral artery was demonstrated left dominant; therefore, we monitored the $\mathrm{rSO}_{2}$ of the brain during operation. By monitoring with INVOS, we could decide whether to use a temporary shunt intraoperatively. Thus, an aorto-right common carotid temporal shunt should be established to ensure safety of the procedure and reduce the risk of cerebral ischemia and infarction. A preoperative knowledge of whether the brachiocephalic artery could be clumped, such as Matas test would be useful.

Conventional treatment for subclavian artery aneurysms is surgical repair and the use of endovascular repair has been increasing. Morbidity and mortality rates of open and endovascular repair are within the same range. ${ }^{3)}$ Vierhout et al. ${ }^{3)}$ described in a review of the literature that the subclavian artery is extremely mobile and exposed to rotational forces during abduction and ante-flexion of the arm. Therefore, a stent-graft has to be flexible and resistant to kinking and fracture. Furthermore, a variety of stent-grafts have been used to date. In-stent stenosis and thrombosis were observed in early series; however, in recent studies, the patency of stent-grafts appears to have increased. Patency results of endovascular treatment may further increase with the development of better stent-grafts and improved postprocedural pharmacotherapy. In our case, the endovascular repair seemed to be difficult. The subclavian artery aneurysm originated just innominate bifurcation. When we consider endovascular repair, the proximal landing zone could not be secured. Resch et al. ${ }^{10)}$ reported a combined open and endovascular approach for such an aneurysm of the innominate bifurcation. They 
achieved to deploy the endograft extending from the innominate into the common carotid artery with a carotidsubclavian bypass and ligation of the subclavian artery distal to the aneurysm. Their reported case was past history of ascending aortic repair combined with valve replacement. In such reoperative and high risk cases, an endovascular repair might be considered.

\section{Conclusion}

We experienced an intrathoracic aneurysm of the proximal part of the right subclavian artery. We consider that entire brachiocephalic artery and right common carotid artery reconstructions are useful when the proximal anastomotic neck cannot be preserved. In cases where it is difficult to anastomose the distal side, it might be useful to close as a stump at just distal side of the aneurysm with aneurismal exclusion and bypass to the distal part of the right subclavian artery.

\section{REFERENCES}

1) Dent TL, Lindenauer SM, Ernst CB, et al. Multiple arteriosclerotic arterial aneurysms. Arch Surg 1972; 105: 338-44. [Medline] [CrossRef]

2) Flores JA, Shiiya N, Nishibe T, et al. Surgical management of aneurysm of the aortic arch vessels and their branches: report of four cases. Surg Today 2003; 33: 917-21. [Medline] [CrossRef]

3) Vierhout BP, Zeebregts CJ, van den Dungen JJ, et al. Changing profiles of diagnostic and treatment options in subclavian artery aneurysms. Eur J Vasc Endovasc Surg 2010; 40: 27-34. [Medline] [CrossRef]

4) Thompson JE. Ligature of the innominate artery for cure of subclavian aneurysms. Ann Surg 1915; 61: 641-57. [Medline] [CrossRef]

5) Caparrelli DJ, Tabulov DM, Freischlag JA. Subclavian artery aneurysm secondary to cervical rib. Arch Surg 2006; 141: 513-4. [Medline] [CrossRef]

6) Clagett GP. Upper extremity aneurysm. In: Rutherford PB, editor. Vascular Surgery. 5th edition. Philadelphia: W.B. Saunders, 2000: 1356-69.

7) Bito Y, Sakaki M, Uji K, et al. Surgical approach to left subclavian artery aneurysm in Marfan syndrome. Gen Thorac Cardiovasc Surg 2009; 57: 376-8. [Medline] [CrossRef]

8) Kawaguchi S, Watanabe M, Hachimaru T, et al. Atherosclerotic pseudoaneurysm of the left subclavian artery: a case report. Ann Thorac Cardiovasc Surg 2010; 16: 376-9. [Medline]

9) Salo JA, Ala-kulju K, Heikkinen L, et al. Diagnosis and treatment of subllavian artery aneurysms. Eur J Vasc Surg 1990; 4: 271-4. [Medline] [CrossRef]

10) Resch TA, Lyden SP, Gavin TJ, et al. Combined open and endovascular treatment of a right subclavian artery aneurysm: a case report. J Vasc Surg 2005; 42: 1206-9. [Medline] [CrossRef] 